\title{
NOTAS
}

\section{LA EXPRESIÓN TEMPORAL EN BERCEO}

Es notable la riqueza de conjunciones temporales que usa Berceo. LANCHETAs, Gramática y vocabulario de las obras de G. de Berceo, pp. $9^{81-}$ 982, sólo enumera las siguientes: quando, mientre, demientre, ante que, luego que, fa(s)ta que, abés, de(s)que, después que; en realidad, Berceo usa otras muchas, la mayoría de las cuales faltan en el Cid.

QUando.-Expresa simultaneidad imperfecta: "Quando fambre avía... oýrme non quisiestes" (Sig, 33a); simultaneidad completa (= 'mientras"): "Ca los evangelistas... quando los escrivien con ella se fablaban" (Mil, 21d). Anterioridad: "De noche, quando era hechado el prior, / issíe por la eglesia fuera de dormitor" (Mil, $79^{b}$ ). Anterioridad inmediata: "Quando rendió el alma el Sennor glorioso / la gloriosa Madre... cadió en tierra muerta" (Duelo, 1oga). Esta última relación temporal se refuerza mediante el adverbio luego: "Quando devota mientre van al su oradero / . . luego da Dios pluvia e sabroso tempero" (SMill, $484 a)$. La anterioridad mediata se expresa también con en cabo quando (='después que"): "En cabo quando fuere leýdo el dictado / aprenderá tales cosas de que será pagado" (SMill, $2 b$ ). Un adverbio suele servir de antecedente a la conjunción: estonz... quando (Mil, 18d), agora... quando (Duelo, 122a), quando... agora (Duelo, 121d). Quando es un verdadero relativo en "Hora quando los omnes façen meridiana" (Oria, $161 \mathrm{c}$ ); lo mismo en al dia quando (Mil, $289 d)$.

Quanto.-No es confusión de quando, como quiere Lanchetas, p. 615. Abunda en el español antiguo: Alex, 730 y 431 (quant); Apol, 155; Sem Tob, 475 (cf. Alarcos, "La lengua de los Proverbios morales de S. T.", $R F E, 35,195^{1}$, p. 305). Equivale a quando en "Pascua fue e grant día quanto tú fuisti nada" (Mil, 79ob). Con luego expresa la anterioridad inmediata (al igual que quando): "Quanto fue acostada fue luego adormida" (Oria, 164a); "quanto ayas el vaso. . . bebido / luego serás connusco" (Martirio, 73a). Equivale a 'mientras' en "Quanto aquí vivimos en ageno moramos" (Mil, 18a). Con igual valor usa Berceo quantos dias ("Quantos dias visquieron", Mil, 404c). La simultaneidad perfecta se expresa también con quanto... en tanto: "Quanto contarie omne poccos de pipiones / en tanto fo tornado cenisa e carbones" ( $M i l$, 372a); el Cid, en este caso, emplea tanto quanto ("tanto quanto yo biua seré dent marauillado", 1038; cf. M. Pidal, I, $\S 1_{53_{3}}$ ); en el Calila aparece ya en tanto que: "en tanto que ellos fueron comer, subió el ximio en çima dela viga" (ed. Allen, Mâcon, ıo6, III, 77). En el Alexandre aparece tanto que para expresar, no la simultaneidad, sino la anterioridad inmediata: "tanto que transponga- 
mos tomarán otro sennor" (ed. Willis, $1848 c=$ 'al punto que, apenas'). Berceo usa con tanto sólo como locución adverbial (Mil, 299a).

Do.-Como quando (Mil, 81 c). Frecuente en español antiguo (cf. M.-Lüвke, III, \$ 597; Hanssen, Gram. hist., \$ 661, y M. Pidal, I, \$ 179.2). Do que = "cuando": "Do que fue a Sant Peydro pareçió la terçera" (Loor, 126a) (¿error por de que?; cf. BogGs, Dictionary of medieval Spanish, p. 195). Usado aún en el Rimado de Palacio: "mucho alegrado era su coraçón donde era atormentado" (1452d), y do (80og).

DE QUE.-Normalmente equivale a 'después que': $D e$ que oraron ellos... tolló Dios a la duenna la mala pestilençia" (SDom, 616a; cf. LANChetas, p. 982). A veces vale por 'desde que': "que avíe muy enfermo, de que morió, guarido" (SMill, $322 d$; cf. Lanchetas, p. 957). Puede tener significado causal: "Sennor, merce te pido de que tanto feçiste / que me non aborrescas quando tant me quisiste" (Loor, $98 c$; cf. M. PIDAL, I, $\S 18_{4_{2}}=$ 'pues que, ya que').

Desque.-Tiene también doble valor temporal: normalmente, 'después que' (Sacr, 188c); reforzado con luego: "Desque faz las tres cruçes, todas son generales, / otras tres faze luego, éssas son speciales" (Sacr, $187 a)$. También equivale a 'desde que': “desque murió la fixa... andaba la su madre por ella fetillada" (Oria, 186a).

Queque.-Tiene, según Lanchetas, p. 984, matiz causal: "Queque foron los otros de muy firme creençia, / tolló Dios a la duenna la mala pestilençia" (SDom, 616b).

Mientre.-Simultaneidad completa. Usada en las formas mientre (Oria, 191a; cf. HANSSEN, $\$ 590)$, mientre que (Duelo, 118a), demientre (solo) y demientre que (SDom, 286a). Berceo no usa mientra(s), forma común en el Cid. Mientre se refuerza con siempre: "Mientre el siglo sea... siempre será contada esta buena fazanna" (SMill, 122c). No expresa propiamente simultaneidad el caso "Deçítmelo demientre avedes la memoria" (Oria, 172d = 'ahora que', 'antes de olvidarlo').

Fasta.-Límite final de la acción. En las cuatro variantes fata ("fata vino el día", SDom, 378a), fata que (Sacr, 4oc), fasta ("non se partieron de elli fasta fue soterrado", Oria, 179d) y fasta que (SDom, 641b). En el Alexandre aparece también ata que (2280).

ANTE oue.-Expresa anterioridad de la oración principal respecto de la temporal: "ante que amanesciesse echáronlis celada" (SDom, $737 b)$. Durante la Edad Media es frecuente la interpolación de palabras entre las dos partes de la conjunción: "Tú ante estás presta que nos te demandemos" (Loor, $217 c)$.

Después Que.-Aunque es frecuente en su tiempo, Berceo sólo la emplea en una ocasión: "quando taiare esa después que se moviere / non fincará los piedes do el sabor obiere" (Duelo, 204c). Prefiere de que, desque, luego que, dessent, o simplemente quando. Sin embargo, usa con relativa frecuencia después solo (como adverbio) o después de: "después de las matinas" (Oria, 26a).

Además de estas conjunciones, usa Berceo otras formas temporales propias de su época: $I$ ) gerundio de simultaneidad: "Iendo en romería caeçi en un prado" (Mil, 2b); 2) expresiones impersonales: ave tanto de tiempo que (SMill, 165), avie luengos tiempos que (Duelo, 1ood), avie 
luengos tienpos passados (Duelo, 101a); 3) la simple coordinación copulativa reforzada con un adverbio temporal: "Acostósse Amunna... $e$ luego ensonnó la su fixa amada" (Oria, $189 c$ = 'apenas', 'en cuanto se acostó'); 4) participio pasado, expresando a) anterioridad amplia: "el meydía pasado..., pidió el cuerpo sancto" (Duelo, $132 a=$ "después de'), b) anterioridad inmediata: "Dichas estas palabras... tollióseli de oios" (Mil, 63a = 'luego que', 'apenas'). La inmediación se refuerza con luego: "Luego las misas dichas, plegóse el conceio" (Mil, 893a), o con inversión, [participio]... luego (Sacr, 165a), o con otra locución temporal: "esta palabra dicha, a poca de sazón / vínoli grant conducho" (SMill, $256 a)$.

Hasta ahora, las locuciones estudiadas son frecuentes en la época (aparecen ya en el Cid). Pero Berceo emplea además otras conjunciones originales $\mathrm{o}$, al menos, poco usadas anteriormente:

Luego Que.-No aparece en el Cid (cf. M. Pidal, I, $\S \S 1553$ y 1573). En Berceo expresa la simple anterioridad ("luego que fue criado, que se podió mandar, / mandólo ir el padre las obeias curiar", SMill, 5 a), o la anterioridad inmediata ("perdonó a Peydro luego que se rependió" Loor, $113 b$; cf. SDom, $4{ }_{23} b$ ). En el segundo caso, la inmediación temporal suele reforzarse mediante la repetición de luego: "Luego que lo entendieron..., el su muy grant pueblo fue luego descosido" (SMill, $\left.45^{1} a\right)$, o con algún otro adverbio de tiempo: luego que..., adahina (Mil, 278a), luego que... manamano (SMill, 176a). A partir de Berceo, esta conjunción es muy frecuente: Alex, 2185; Apol, 1296; J. Ruiz, 647 , etc.

Assí сомmo.-Acciones inmediatamente sucesivas: "Assi commo lo ouo de la boca passado, / la duenna fo guarida" (SDom, 308a). Usado ya en el Cid: "assi commo entraron, al Cid besaron le las manos", 153 (cf. M. LüBKe, III, $\S \S 594-595$, y Cuervo, Dicc., I, 699).

Соммо.-Equivale a 'mientras' en "Commo asmaba Oria a su entendimiento, / oió fablar a Christo" (Oria, 88a). Compárese: "E commo yuan aserrando metían vna cuña e sacauan otra por aserrar mejor" (Ca. lila, III, 75). No obstante, como suele expresar la anterioridad inmediata, no la simultaneidad: "todo onbre como un amor pierde luego otro cobre" (J. Ruiz, 159; cf. BogGs, p. $126=$ 'en cuanto'). Con este valor es frecuente en la lengua clásica (Keniston, 28.56 y 29.811; en el Quijote, ed. R. Marín, t. 1, p. 399, n.4).

Commo Que.-"La freyra con la pérdida que avíe reçibida / issió como que pudo ont iaçíe escondida" (Mil, 888b). A. Alonso ("Español como que y cómo que", RFE, 12, 1925, p. 137) parece considerarla modal; no obstante, el sentido pide más bien 'luego que, apenas' (cf. BogGs, p. 126: "como que, en cuanto").

ABÉs, AvÉs.-Anterioridad inmediata: “Avés avía don' Oria el vierbo acabado, / plegó la Gloriosa" (Oria, 122a; además, Mil, 476a y 596a). LANChetas, p. 119 y Corominas, DCELC, s. v., se refieren sólo a su valor adverbial, 'difícilmente'. Como conjunción temporal se encuentra tam. bién en Alex, 177 y otros textos. La combinación aun abés, en Alex, 12: "aun abés faulaua ya lo yuan temiendo".

Aún No.-Con igual valor: "Aún del monesterio non eran alonga- 
dos, / fueron mal confondidos por sos graues pecados" (SMill, 273a). Se encuentra también en el Fernán González: "Non podrýe el buen conde azn ser byen dormido, / el monje San Pelayo de súsol fue venido" (403a). No bien es construcción clásica (cf. García de Diego, Gram. hist., p. 376).

MAN A MANO QUF.-Expresa también sucesión inmediata: "Man a mano que fuy en tierra acostado, / de todo el lazerio fui luego folgado" (Mil, 12a). Como adverbio temporal lo usa Berceo más veces: "Fiz el omne bueno man a mano su yda" (Martirio, 61c = 'inmediatamente'); "Fusó la maletía del cuerpo manamano" (SMill, 13oc; cf. $176 b$ y $328 b$ ). Solalinde (ed. de los Milagros en Clás. cast., p. 4) cita dos casos de man a mano (de la Razón de amor, 124, y de la Estoria de los cuatro dotores, p. 46), pero sólo como adverbio temporal (= 'en seguida'), no como conjunción. Tampoco Lanchetas repara en su valor conjuntivo.

Adiesso Que.-Con igual significado que las anteriores: "Adiesso que Teófilo... reçibió Corpus Domini, ...fue... de claridat cercado" (Mil, 85oa); "adiesso que ovieron las ganancias partidas" (SMill, 459a). Lanchetas, p. 128 , sólo habla de su función adverbial: "La yent más liviana adiesso fue venida" (Mil, 888d). No lo encuentro en otras obras del siglo xiII.

Que.-Como quando: "Quando vino a ellos, que fue en la ribera, / conocieron lo todos que el que salió era" (Mil, 6o5a). ¿Será omisión de la preposición de en "de que"?

Precedida de un pronombre personal, la conjunción que tiene con frecuencia valor temporal (simultaneidad): "Ellos que la levaban non de buena manera, / violo Sanctiago, cuyo romero era" (Mil, 198a); "ellos que se querien todos ir su carrera, / estendieron los oios... vidieron" (Mil, 442a). Cf.: "et traýa vos vna liebre que vos enbiauan que yantásedes. Et yo que venía çerca [de aqui], falló me vn león e tomó mela" (Calila, III, 551). Aparece todavía en Mena, como arcaísmo (cf. M. R. Lida de Malkiel, Juan de Mena, México, 1950, p. 293). En la lengua clásica, con el neutro 10 : "no perderé vuestra amistad en cuanto la vida me durare, y quisiera ser natural destos remos por estar más cercano a vuestro servicio; pero lo que en ellos asistiere..., eso me tendréis muy pronto a serviros" (= 'mientras'; Castillo Solórzano, La Garduña de Sevilla, en Clás. cast., p. $\left.161_{31}\right)$. En que, por 'cuando', no usado en Berceo, se encuentra ya en el Calila: "Et los otros sus compañeros, en que lo vieron asý, fuyeron" (II, 174; cf. III, 278).

A LA HORA DE + imfinitivo.-Vale por 'cuando'; frecuente en Berceo: "Díssolis a la ora de la alma essir" (SMill, 299a); "que non nos desconoscan a la hora de entrar" (Loor, 167d); "Diohs un mal espanto a la hora dél prender" (Loor, 59a). Nada dice LANCHETAs. Su origen puede estar en las construcciones ser o estar en hora de: "Madre, agora somos en ora de rancar" (Duelo, 89a); "que en hora estaban de la alma echar" (Duelo, $\left.{ }_{15} 8 d\right)$; esta última es perífrasis incoativa $=$ 'a punto de'. He encontrado la misma construcción en otras obras medievales: "acostado a un pilare / como se acostó a la hora de finare" (Roncesvalles, 29); "e guarden al hora del arrencar que los arranquen con sus rraýses" (Tratado de agri. cultura de Ibn Bassal, en $A l A n, 13,1948$, p. $3^{847}$ ); o con verbo finito: 
"ca el trasponer les fase mal e enflaquéçelos a la hora que los arrancan" (Trat. de agric., p. 382 $32_{32}$ ). Cf. la conjunción alora que usada desde el Cid: "al ora que lo sopo myo Cid... plógol de coraçón" (v. 1454); "tomaua el rosinor enla mano, et ala ora que beýa la çigunnela trauáual dela pierna" (D. Juan Manuel, Libro de la caza, ed. Castro y Calvo, p. 99:); ejemplos clásicos en Keniston, Syntax, 28.56 .

AL + infmitivo.--Berceo es el primero que usa esta construcción, no muy frecuente durante la Edad Media: "Al posar, al mover todas se esperavan" (Mil, 8c; cf. Lanchetas, Pp. 953 y 998). Otros ejemplos medievales: "al sacar dela cuña apretó la viga" (Calila, III, 79); "muy grant pesar ouieron al partir" (Cauallero Zifar, ed. Wagner, p. 10514); "al tomar te alegras" (J. Ruiz, 246b).

Participio PRESENTE.-Forma todavía oraciones temporales, expresando simultaneidad: "Entrante de la eglesia..., comediólo la tercera vegada" (Mil, 473a), o anterioridad: "un omne bueno que perdiente el viso" (Martirio, 59 ).

El TIEMPo QUE.-Precedida de preposición, forma diversas locuciones temporales; Berceo usa al tiempo que y en el tiempo que para expresar simultaneidad: "Al tiempo que Valerio tenía la bispalía, / nudrió estos criados" (Martirio, 3a); "ennos tiempos derechos que corría la verdad..., estonz vivíen a buenas, vinien a vegedat" $(M i l, 502 a)$. En los siglos posteriores se usaron varias locuciones formadas con la voz tiempo: "todo aquel tiempo que estouieron en su obediencia, gozauan de paz" (= 'mientras', Pulgar, Claros varones, en Clás. cast., 17.4); en el tiempo que (Pulgar, 49, Corbacho, ed. Simpson, 2196); a tiempo que (= 'cuando', Pulgar, $110_{4}$ ); el tienpo que (='mientras', Corbacho, 29. $)$; al tienpo que (= 'cuando', Corbacho, 10410); en tienpo que (DIEGo DE San Pedro, Cárcel de amor, en Clás. cast., 125i), etc.

EL día QUE.-Berceo no la usa propiamente como conjunción ("viernes era el dia que esto conteçió", SMill, 379a), pero construcciones de este tipo originaron la locución temporal verdaderamente conjuntiva: "del dia que fue conde" (Cid, 1062 = 'desde que'; cf. M. Pidal, I, $\S 155_{3}$ ); "del dia en que nasci" (Zifar, 160...); "non las deuen meter en agua synon el dia que obran con ellas" (= 'cuando', Trat. de agric., $405 \%$ ).

SIEMPRE QUANDo.--Quizá pueda considerarse verdadera conjunción temporal en "Siempre quando queríes a Dios sacrificar, / queríes la sancta missa deçir en el altar" (Martirio, 65a), ya que Berceo no usa cada que nunca, aunque cada, solo, "expresa idea de totalidad [= "todos'] con sentido distributivo" (cf. Lanchetas, pp. 191-192): "esto es cada día" (Mil, joa). Cada que tampoco aparece en el Cid, pero sí en el Calila: "Et cada que sacaua los pollos comíagelos la culebra" (III, 461).

Otra conjunción que falta en Berceo es pues (que), usada ya en el Cid: "pues esto an fablado, pienssan se de adobar" (v. 1283; cf. M. Pidal, I, \$195); "pues que a Casteión sacaremos a çelada" (Cid, 441); "pues naçi non ui tan bella" (Razón de amor, 57; = "desde que', lo mismo que desque o después que); "ella misma se quema pues que es mediada" (Alex, 2311). Berceo sólo usa pues como adverbio: "nin pues nin ante non ovo compannera" (Sacr, $5^{8 b}$ ). 
SóLo QUE.-Puede advertirse cierto valor temporal en la conjunción sólo que, normalmente condicional: "Doquiere que la tenga el diablo metida, / sólo que Él lo quiera luego será rendida" $\left(M i l, 8 \mathbf{o}_{4} b\right)=$ 'en cuanto, luego que'. Lo mismo en SMill, 403 d, y Loor, 60 a (cf. LancheTas, p. 892: sólo que $=$ 'luego'). Exclusivamente temporal es en estos dos casos: "Ha me deparado mi ventura vna gulpeja, et sol que sabe que mis palominos son criados viéneme amenazar" (Calila, XVI, 18); "Mas agora, sennor, ha menester que sol que vos uenga gente de Mayorgas e de las otras yslas, que me acordades por que non sea conquerido de tan uil gente" (Don Juan Manuel, Documentos, ed. Giménez Soler, $318_{19}$ ).

Es de dudosa interpretación el dessent quando de San Millán (221a): "Dessent quando ovieron echados los tizones / prisiéronse a pelos e a los cabezones". ¿Será dessent, quando (= 'después, cuando...') o dessent quando (= 'después que')? Compárese, en el Cid, de quando = 'desde que': "ca de quando nasco adeliçio fue criada" (3284; cf. M. PIDAL, II, p. 819).

E1 Colegio de México.

Juan M. Lope Blanch

\section{EL MISTERIOSO ORIGEN DE FERNÁN GONZÁLEZ}

En un reciente artículo sobre las mocedades de Fernán González, don Ramón Menéndez Pidal ha hecho notar cuán escasa es la documentación sobre los primeros años del famoso Conde batallador. Su madre, Munniadonna, enviudó del rey García I en 914. Hijo de un segundo matrimonio, el héroe no pudo, pues, nacer antes de 915. Nada sabemos del joven hasta 929, año en que se firmó "Conde de Lara"; tenía entonces, a lo más, catorce años. En 933, ya "Conde de Castilla", estaba casado con doña Sancha de Navarra1. Doña Sancha era hija del difunto rey Sancho, hermana del rey García Sánchez y de doña Urraca, reina de León. No nos sorprende que el Conde, emparentado con personajes de alta alcurnia, desempeñara un papel tan importante en los turbulentos días de la naciente Castilla.

Tampoco nos sorprende que la epopeya castellana, tan aficionada al mito, atribuyese al Conde hazañas sin par. Para hacer más impresionantes las proezas del héroe, el desconocido monje de Arlanza que compuso el Poema de Fernán González acudió al contraste: al presentar por vez primera al joven Fernán, nos lo muestra sometido a la tutela de un "pobrezyello que labraua carbón", viviendo, pues, en condiciones ínfimas. Los gloriosos hechos que realiza después y las alturas a que asciende se destacan de manera inolvidable sobre el fondo oscuro de la primera visión que de él tenemos.

Ahora bien, dice Marden en la Introducción a su edición crítica del Fernán González ${ }^{2}$ que, fuera del Poema, no ha encontrado "otra men-

1. R. Menéndez PrdAL, "Fernán González, su juventud y genealogia", $B A H, 134$ $(1954), 335-358$

2 C. Carroll Marden, Poema de Fernán González. Texto crítico con introducción, notas y glosario, Baltimore, 1904, p. 175 . 